\title{
EFFICIENCY ISSUE IN C-BAND KLYSTRON-MODULATOR SYSTEM FOR LINEAR COLLIDER
}

\author{
J.S. Oh, M.H. Cho, W. Namkung, T. Shintake ${ }^{1}$, H. Matsumoto ${ }^{1}$, K. Watanabe ${ }^{2}$, and H. Baba ${ }^{3}$ \\ PAL/POSTECH, Pohang, Kyungbuk, 790-784, Korea \\ ${ }^{1}$ National Laboratory for High Energy Physics, Tsukuba, 305, Japan \\ ${ }^{2}$ Laboratory of Nuclear Science, Tohoku University, Sendai, 982, Japan \\ ${ }^{3}$ Nihon Koshuha Co., Ltd, Yokohama, 226, Japan
}

Abstract

A C-band $(5,712 \mathrm{MHz}) \mathrm{RF}$ system for an e+e- linear collider at $500 \mathrm{GeV}$ center-of-mass energy requires total 4,080 units of $50 \mathrm{MW}$ pulse klystrons and matching 111 MW peak-power modulators for two main linacs. An initial design value of the wall plug power for the entire RF system is $150 \mathrm{MW}$ with $2.5 \mu$ sec RF pulse width and $100 \mathrm{~Hz}$ pulse repetition rate. A detailed circuit analysis shows that it is possible to get the overall RF system efficiency of approximately $24 \%$, or equivalent to 210 MW wall plug power. This includes $85 \%$ DC high voltage charging efficiency using compact inverter power supplies, and $70 \%$ pulse efficiency using $3.5 \mu$ sec pulse length (ESW) with less than $1 \%$ flat top ripple, and auxiliary powers for thyratron heater, klystron heater, focusing magnet, pulse transformer reset, and etc.

\section{INTRODUCTION}

An e+e- linear collider at $500 \mathrm{GeV}$ C.M. energy is a very large scale machine of which total length is around $20 \mathrm{~km}$. There are many general requirements for this system such as high reliability and availability, reasonable power efficiency, lower construction cost, simple and easy maintenance, easy and flexible operation.

This paper analyzes the power consumption and efficiency of a C-band RF system for an e+e- linear collider at $500 \mathrm{GeV}$ C.M. energy. This system has total 4,080 units of klystrons (50 MW peak) and modulators (111MW peak) for two main linacs[1].

\section{SYSTEM DESCRIPTION}

Table 1 shows specifications of a C-band RF system including a klystron and a modulator. A modulator produces $3.5 \mu \mathrm{sec}$ (ESW, Equivalent Square Wave) pulse with $111 \mathrm{MW}$ peak power. The flat-top portion of 2.5 $\mu$ sec with ripple less than $1 \%$ is utilized to generate 50 MW RF power by a klystron with $45 \%$ conversion efficiency. Fig. 1 is a simplified circuit diagram of the modulator. The HV inverter power supply provides DC high voltage generation with $0.5 \%$ fine regulation of $\mathrm{PFN}$ charging voltage without de-Qing system. It also has a feature of command charging. This makes the system simple, modular, and reliable. A PFN is a 16-stage Guillemin E-type LC network. There is no thyrite to present the low inverse voltage required for the thyratron deionization in the EOLC circuit due to the command charging scheme of the inverter power supply.

Table 1: Specifications of a C-band RF system.

\section{Klystron (E3746)}

Operating frequency

Peak output power

Repetition rate

Efficiency

Perveance

Gun voltage

Beam current

RF pulse length

Modulator

Peak output power

Average output power

HV pulse length

Peak switching current PFN charging voltage

PFN impedance

Stored energy in PFN

PFN cell number

$$
\begin{array}{rl}
5712 & \mathrm{MHz} \\
50 & \mathrm{MW} \\
100 & \mathrm{pps} \\
45 & \% \\
1.53 & \mu \mathrm{A} / \mathrm{V}^{1.5} \\
350 & \mathrm{kV} \\
317 & \mathrm{~A} \\
2.50 & \mu \mathrm{sec}
\end{array}
$$

111 MW

$38.9 \mathrm{~kW}$

$3.50 \mu \mathrm{sec}(\mathrm{ESW})$

$4758 \mathrm{~A}$

$46.7 \mathrm{kV}$

$4.91 \Omega$

389 Joule

16

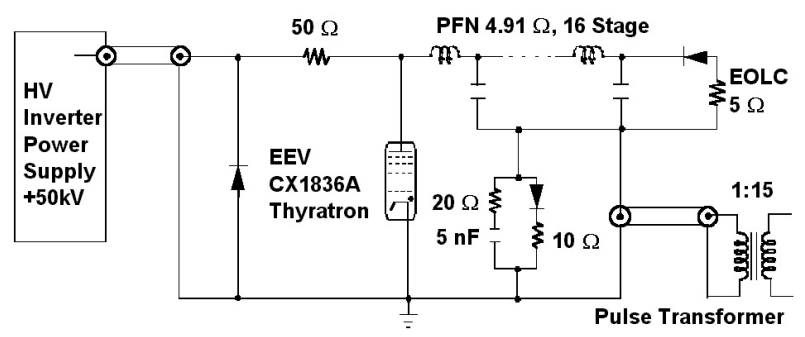

Figure 1: Circuit schematic of a C-band modulator.

\section{DEFINITION OF EFFICIENCY}

Fig. 2 is a block diagram of power flow for a pulsed RF system. It shows the power conversion flow and corresponding efficiencies of each sub-system. A charging system with $\mathrm{P}_{\mathrm{AC}}$, input power supplies $\mathrm{P}_{\mathrm{DC}}$, charging power to PFN of a pulsing system. A pulsing system produces $\mathrm{P}_{\text {PULSE, }}$ pulse power which is the flat-top portion of a pulse. Then a klystron utilizes it to generate $\mathrm{RF}$ power, $\mathrm{P}_{\mathrm{RF}}$. A $\mathrm{P}_{\mathrm{AUX}}$, auxiliary power includes the solenoid coil power for klystron magnet, heater powers for thyratron and klystron, a core reset power for a pulse transformer, a cooling fan power, and so on. $\eta_{\mathrm{C}}, \eta_{\mathrm{P}}$, and $\eta_{\mathrm{K}}$ represent efficiencies of each sub-system. Table 2 
is the list of essential efficiencies.

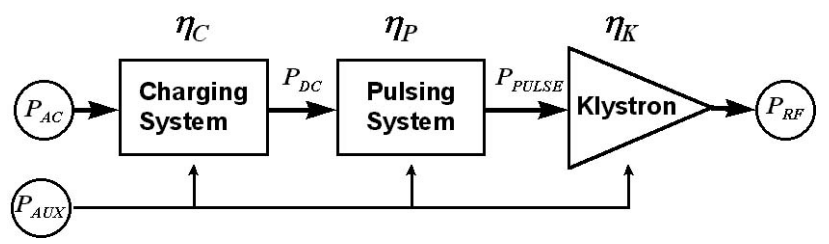

Figure 2: Power flow and efficiencies of an RF system.

Table 2: Definition of efficiencies.

$$
\begin{array}{ll}
\eta_{\mathrm{C}}=\mathrm{P}_{\mathrm{DC}} / \mathrm{P}_{\mathrm{AC}} & ; \text { Charging Efficiency } \\
\eta_{\mathrm{P}}=\mathrm{P}_{\mathrm{PULSE}} / \mathrm{P}_{\mathrm{DC}} & ; \text { Pulsing Efficiency } \\
\eta_{\mathrm{K}}=\mathrm{P}_{\mathrm{RF}} / \mathrm{P}_{\mathrm{PULSE}} & ; \text { Klystron Efficiency } \\
\eta_{\mathrm{M}}=\mathrm{P}_{\mathrm{PULSE}} / \mathrm{P}_{\mathrm{AC}} & ; \text { Modulator Efficiency } \\
\eta_{\mathrm{KM}}=\mathrm{P}_{\mathrm{RF}} / \mathrm{P}_{\mathrm{AC}} & ; \text { Klystron-Modulator Efficiency } \\
\eta_{\mathrm{RF}}=\mathrm{P}_{\mathrm{RF}} /\left(\mathrm{P}_{\mathrm{AC}}+\mathrm{P}_{\mathrm{AUX}}\right) & ; \text { RF System Efficiency }
\end{array}
$$

\section{RISE TIME ANALYSIS}

A charging system has an efficiency of $85 \%$ specified by the vendor of the inverter power supply. The efficiency of a klystron is given by the design value of $45 \%$. A pulse efficiency depends on the detail design parameters of the pulsing system including a pulse transformer and a klystron. The rise time of a pulsing system including a pulse transformer and a klystron load for a matched PFN can be described by following equations;

$$
\begin{aligned}
& \boldsymbol{T}_{\boldsymbol{r}}=\boldsymbol{f}(\boldsymbol{\sigma})\left(\left(\boldsymbol{L}_{W}+\boldsymbol{L}_{\boldsymbol{L}}\right)\left(\boldsymbol{C}_{\boldsymbol{D}}+\boldsymbol{C}_{\boldsymbol{L}}\right)\right)^{0.5} \\
& f(\sigma)=2 \sigma(m /(m+1))^{0.5}\left(0.255-0.256 \sigma+0.537 \sigma^{2}\right) \\
& \sigma=(\gamma m+1 / \gamma) /\left(2(m(m+1))^{0.5}\right) \\
& m=R_{L} / R_{G} \\
& \gamma=Z_{T} / R_{L} \\
& Z_{T}=\left(\left(L_{T}+L_{W}\right) /\left(C_{D}+C_{L}\right)\right)^{0.5}
\end{aligned}
$$

where $f(\sigma)$ is a fitting function to give the rise time from $10 \%$ to $90 \%$ of maximum pulse height, $L_{L}$ is the leakage inductance of a pulse transformer, $L_{W}$ is the wiring inductance of a system, $C_{D}$ is the distributed capacitance of a pulse transformer between primary and secondary coils, $C_{L}$ is the distributed load capacitance of a system including the klystron, $R_{L}$ is the klystron load impedance, $R_{G}$ is the PFN generator impedance. $Z_{T}$ is the transformation impedance of the pulse transformer including $L_{W}$ and $C_{L}$, and $m$ is the matching parameter between the PFN and the klystron. $\gamma$ is the impedance matching parameter between the transformation system and the klystron.

For a lumped PFN modulator with given total distributed capacitance and total leakage inductance, the optimum value of $f(\sigma)$ for generating good waveforms is 1.74 with $\sigma$ of $0.78, \gamma$ of 1.58 , and $m$ of 1.05 . A good waveform means that it has a minimum rise time with less than $2 \%$ overshoot. A larger $\gamma$ gives a slow rise time and a smaller $\gamma$ makes the large overshoot with ringing. It is important to keep the optimum $\gamma$, and minimize distributed capacitance and leakage inductance to get the minimum rise time with good waveforms. A wiring inductance of $1 \mu \mathrm{H}$ of the pulsing system at the primary side with 15 step-up ratio of the transformer gives $225 \mu \mathrm{H}$ inductance at the secondary side. This inductance corresponds to the minimum rise time of $0.22 \mu$ s with $\gamma$ of 1.58. Fig. 3 is the analyzed result of distributed capacitances using DENKAI in the pulse tank including the klystron assembly. The right-half of the figure shows potential lines in the region of the klystron tube and between the coils of the pulse transformer in the tank. The calculated values of capacitance are presented at the left-half of the figure. Total distributed capacitance in the tank except the capacitance between the coils is $115 \mathrm{pF}$. If the capacitances between the corona ring and other structures not included in this estimation is added to the result, it becomes approximately $120 \mathrm{pF}$. This capacitance corresponds to the minimum rise time of 0.39 $\mu$ with $\gamma$ of 1.58. So the large distributed capacitance in the pulse tank is more limiting parameter for the pulse rise time in the case of the C-band RF system.

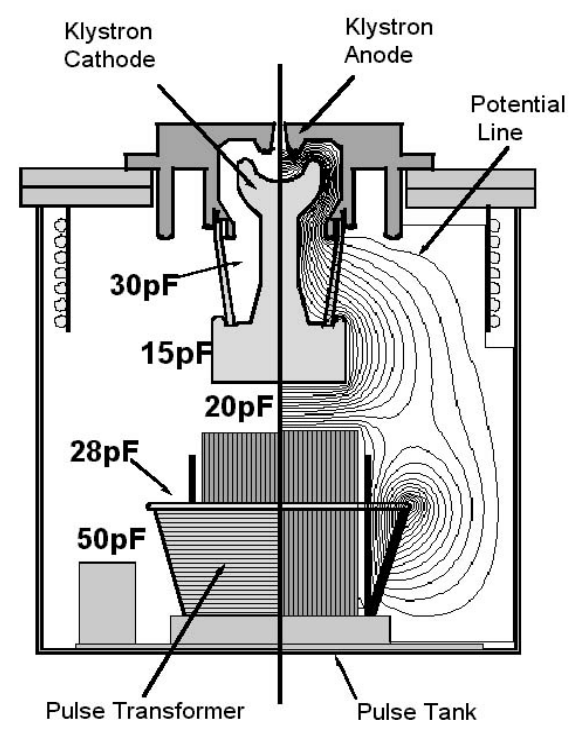

Figure 3: Distributed capacitance and potential lines in the pulse tank with E3746 klystron.

Table 3: Parameters of the C-band pulse transformer.

$\begin{array}{lr}\text { Turn Ratio } & 15 \\ \text { Primary turns } & 3 \\ \text { Leakage inductance }(\mu \mathrm{H}) & 1.60 \\ \text { Stray capacitance }(\mathrm{nF}) & 6.33 \\ \text { Shunt Inductance }(\mathrm{mH}) & 0.36 \\ \text { Core Resistance }(\Omega) & 411 \\ \text { Droop }(\%) & 2.39 \\ \text { Overshoot }(\%) & 1.96\end{array}$

The primary consideration of the transformer design is to keep the capacitance low between primary and secondary coils, for example under $30 \mathrm{pF}$ to make the rise time of less than $0.5 \mu$ s and guarantee the flat-top width of $2.5 \mu \mathrm{s}$. Typical parameters of the pulse transformer at the primary side are summarized in Table 3 . Total inductance 
and capacitance including transformer parameters are 499 $\mu \mathrm{H}$ and $164 \mathrm{pF}$ which give the rise time of $0.47 \mu \mathrm{s}$.

\section{POWER DISTRIBUTION ANALYSIS}

The simulated klystron voltage waveform with its expanded view using PSPICE is shown in Fig. 4. The expanded view shows $2.5 \mu$ s flat-top with ripples less than $1 \%$ which results the phase shift of $5.68^{\circ}$ of the klystron RF output. Analyzed power distribution of a C-band klystron-modulator system is described in Table 4. Total $51.4 \mathrm{~kW}$ is required to generate $12.5 \mathrm{~kW}$ RF power with $100 \mathrm{~Hz}$ operating condition. The beam power of $15.3 \mathrm{~kW}$ is dumped to a collector of a klystron within the portion of flat-top. This loss caused by the conversion inefficiency of the klystron is $30 \%$ of the total power. Wasted pulse power includes all the losses which are not contributed to make RF power from the stored energy of the PFN. The loss during rise and fall time is $10 \mathrm{~kW}$ which includes stored energies in the leakage inductance and the distributed capacitance, and electron beam power delivered to the collector during this time interval. This is the main loss in the pulse modulator, which is $19.5 \%$ of the total power. The magnetizing loss of $0.9 \mathrm{~kW}$ means the stored energy in the shunt inductance of the pulse transformer which is finally dissipated at the tail clipper. The solenoid coil power of the klystron magnet is assumed just as $3 \mathrm{~kW}$ but it still takes main part of the auxiliary power.

Table 4: Power distribution of a C-band system.

\section{Total Power $=\mathbf{5 1 , 3 7 1 ~ W}$}

1. RF Power

2. Wasted Beam Power

$\begin{array}{rr}12,488 & (24.3 \%) \\ 15,263 & (29.7 \%) \\ 12,071 & (23.5 \%) \\ 7,072 & (13.8 \%) \\ 4,477 & \left(\begin{array}{r}8.7 \%)\end{array}\right.\end{array}$

3. Wasted Pulse Power

4. Charging Loss

5. Aux. Power

Wasted Pulse Power

a. Rise/Fall Time Loss

b. Magnetizing Loss

c. Thyratron Loss

d. Eddy Current Loss

e. RC Snubber Loss

Aux. Power
a. Klystron Magnet
b. Thyratron Heater
c. Cooling Fan
d. Klystron Heater
e. Core Bias
f. Thyratron Reservoir
$3,000(5.84 \%)$
$567(1.10 \%)$
$450(0.88 \%)$
$316(0.62 \%)$
$100(0.19 \%)$
$44(0.09 \%)$

9,991 (19.45\%)

929 ( $1.81 \%)$

$416(0.81 \%)$

$463(0.90 \%)$

$272(0.53 \%)$

\section{EFFICIENCY}

Table 5 shows the efficiencies of the each sub-system of the C-band klystron-modulators. The pulse efficiency of $70 \%$ is mainly limited by distributed capacitance in the oil-filled pulse tank. It is difficult to improve the pulse efficiency using the standard method. Since a net RF system efficiency is $24 \%$, the total AC wall-plug power

of $210 \mathrm{MW}$ will be consumed to generate the total RF power of 51 MW using 4,080 units of a klystronmodulator system.

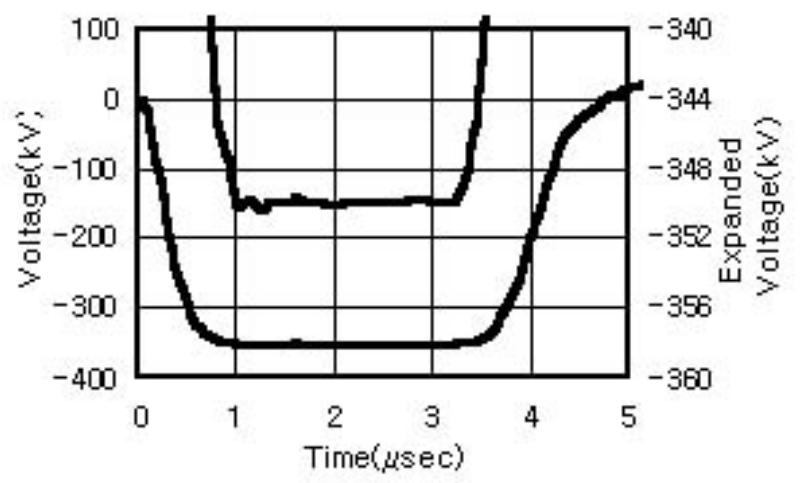

Figure 4: Simulated klystron voltage waveform.

\begin{tabular}{lr} 
Table 5: Efficiencies of the C-band system. \\
\hline Charging Efficiency & $85.0 \%$ \\
Pulse Efficiency & $69.8 \%$ \\
Klystron Efficiency & $45.0 \%$ \\
Modulator Efficiency & $59.3 \%$ \\
K-M Efficiency & $26.7 \%$ \\
RF System Efficiency & $24.4 \%$
\end{tabular}

\section{CONCLUSIONS}

A net RF efficiency of the C-band system is estimated to be $24 \%$, and this requires total $\mathrm{AC}$ wall-plug power of 210 MW. The charging efficiency of $85 \%$ can be improved up to $90 \%$ through the trade-off design of the inverter power supply. The pulse efficiency of $70 \%$ is almost optimized. If the PM-AM modulation method of the C-band system[2] allows the 2-3\% tolerance of the flat-top ripple, it can be improved up to $75 \%$. The klystron efficiency of $45 \%$ is the most flexible parameter in the RF sub-systems. It could be raised up to $60 \%$. Then it is expected to get a net RF efficiency of $35.4 \%$, and total AC wall-plug power demand can be lowered to $144 \mathrm{MW}$.

\section{REFERENCES}

[1] Shintake, et al., C-band Main Linac RF System for e+e- Linear Collider of 0.5 To 1.0 TeV C.M. Energy, Proc. $18^{\text {th }}$ International Linear Accelerator Conference, Geneva, Switzerland, August 26-30, 1996.

[2] T. Shintake and N. Akasaka, A New RF PulseCompressor Using Multi-Cell Coupled-Cavity System, Proc. $5^{\text {th }}$ European Particle Accelerator Conference, Sitges, Barcelona, Spain, June 10-14, 1996. 